\title{
Human Motor Corpus Callosum: Topography, Somatotopy, and Link between Microstructure and Function
}

\author{
Mathias Wahl, ${ }^{1}$ Birgit Lauterbach-Soon, ${ }^{1}$ Elke Hattingen, ${ }^{2}$ Patrick Jung, ${ }^{1}$ Oliver Singer, ${ }^{1}$ Steffen Volz, ${ }^{3}$ \\ Johannes C. Klein, ${ }^{1}$ Helmuth Steinmetz, ${ }^{1}$ and Ulf Ziemann ${ }^{1}$ \\ ${ }^{1}$ Department of Neurology, ${ }^{2}$ Institute of Neuroradiology, and ${ }^{3}$ Brain Imaging Center, Johann Wolfgang Goethe-University of Frankfurt, D-60528 Frankfurt \\ am Main, Germany
}

The corpus callosum (CC) is the principal white matter fiber bundle connecting neocortical areas of the two hemispheres. Although an object of extensive research, important details about the anatomical and functional organization of the human CC are still largely unknown. Here we focused on the callosal motor fibers (CMFs) that connect the primary motor cortices (M1) of the two hemispheres. Topography and somatotopy of CMFs were explored by using a combined functional magnetic resonance imaging/diffusion tensor imaging fiber-tracking procedure. CMF microstructure was assessed by fractional anisotropy (FA), and CMF functional connectivity between the hand areas of M1 was measured by interhemispheric inhibition using paired-pulse transcranial magnetic stimulation. CMFs mapped onto the posterior body and isthmus of the CC, with hand CMFs running significantly more anteriorly and ventrally than foot CMFs. FA of the hand CMFs but not FA of the foot CMFs correlated linearly with interhemispheric inhibition between the M1 hand areas. Findings demonstrate that CMFs connecting defined body representations of M1 map onto a circumscribed region in the CC in a somatotopically organized manner. The significant and topographically specific positive correlation between FA and interhemispheric inhibition strongly suggests that microstructure can be directly linked to functional connectivity. This provides a novel way of exploring human brain function that may allow prediction of functional connectivity from variability of microstructure in healthy individuals, and potentially, abnormality of functional connectivity in neurological or psychiatric patients.

Key words: motor corpus callosum; diffusion tensor imaging; fractional anisotropy; microstructure; transcranial magnetic stimulation; interhemispheric inhibition; functional connectivity

\section{Introduction}

The corpus callosum (CC) is the principal white matter fiber bundle connecting neocortical areas of the two hemispheres (Innocenti, 1986; Gazzaniga, 2000). Although it is an object of extensive research, important details about the anatomical and functional organization of the human CC are still largely unknown. This pertains to the callosal motor fibers (CMFs) connecting the primary motor cortices (M1) in the two hemispheres. Anatomical studies in the rhesus monkey located the CMFs into the anterior half of the body of the CC (Pandya and Seltzer, 1986; Schmahmann and Pandya, 2006), and these fibers seem to be somatotopically organized (Schmahmann and Pandya, 2006). In contrast, evidence from transcranial magnetic stimulation (TMS) (Meyer et al., 1998) and two recent diffusion tensor imaging (DTI) studies (Hofer and Frahm, 2006; Zarei et al., 2006) suggested that the CMFs in humans run in the posterior body of the CC. However, detailed data about CMFs in the human CC are

\footnotetext{
Received May 21, 2007; revised Sept. 4, 2007; accepted Sept. 5, 2007.

This work was supported by an educational grant from TEVA Pharmaceuticals. We thank George Ogrezeanu for technical help and Ralf Deichmann for support with the revision of this manuscript.

Correspondence should be addressed to Prof. Ulf Ziemann, Motor Cortex Group, Department of Neurology, Johann Wolfgang Goethe-University of Frankfurt, Schleusenweg 2-16, D-60528 Frankfurt am Main, Germany. Email: u.ziemann@em.uni-frankfurt.de.

DOI:10.1523/JNEUROSCI.2320-07.2007

Copyright $\odot 2007$ Society for Neuroscience $\quad$ 0270-6474/07/2712132-07\$15.00/0
}

still limited, and it is unknown if they are somatotopically organized.

It is a long-held view that the motor CC is important for bimanual coordination and learning of bimanual motor skill. This notion was built mainly on bimanual coordination deficits observed in patients with extensive lesions of the CC or partial callosotomies (Preilowski, 1972; Jeeves et al., 1988; Andres et al., 1999; Eliassen et al., 1999, 2000; Wiesendanger and Serrien, 2004; Caille et al., 2005). One step toward a refined understanding of the function of the human CC would be to identify its detailed topographical organization by novel MRI techniques. DTI allows quantification of the directionality of diffusion by the fractional anisotropy (FA) index. FA estimates the orientational coherence of white matter fiber bundles (Moseley et al., 1990; Le Bihan et al., 2001; Beaulieu, 2002; Le Bihan, 2003; Pfefferbaum et al., 2003). Combining DTI with functional magnetic resonance imaging (fMRI) allows noninvasive quantitative in vivo analysis of anatomical connections between functionally defined brain regions (Conturo et al., 1999; Koch et al., 2002; Dougherty et al., 2005; Kim and Kim, 2005). Regional FA of subcortical association fibers correlated topographically specifically with cognitive functions such as reading ability (Klingberg et al., 2000), visuospatial attention (Tuch et al., 2005), or mental object rotation (Wolbers et al., 2006), and FA of the total CC correlated with visuomotor interhemispheric processing speed (Schulte et al., 2005). This 
introduces a new window to study the brain, with regional FA serving as a microstructural surrogate marker that links directly to function. Functional connectivity between the M1 hand areas of the two hemispheres can be assessed objectively by a pairedpulse TMS protocol that measures transcallosally mediated interhemispheric inhibition (IHI) (Ferbert et al., 1992; Di Lazzaro et al., 1999).

This study aims at reexamining by combined fMRI/DTI-fiber tracking the topography of CMFs in the human CC and at providing novel information as to whether CMFs of different body representations are somatotopically organized. Another main purpose is to clarify to which extent CMF microstructure, as measured by FA, relates to functional connectivity of these fibers, as assessed by IHI.

\section{Materials and Methods}

Subjects. Twelve healthy right-handed subjects (mean age, $32.6 \pm 9.0$ years; 5 female) were tested. Written informed consent to participate in the study was obtained from all subjects. The study was approved by the local ethics committee of the University Hospital of Frankfurt/Main.

Image acquisition. Magnetic resonance imaging was performed on a 3 tesla Magnetom Trio (Siemens Medical Solutions, Erlangen, Germany): structural T1-weighted data were acquired using a magnetizationprepared rapid-acquisition gradient echo (MPRAGE) sequence [repetition time (TR) $2250 \mathrm{~ms}$, echo time (TE) $26 \mathrm{~ms}$, inversion time $900 \mathrm{~ms}$, flip angle $9^{\circ}, 192$ sagittal slices, field of view $256 \times 256 \mathrm{~mm}$, voxel size $1 \times$ $1 \times 1 \mathrm{~mm}$ ). To localize body representations (lip, hand, foot) in the M1 of either hemisphere, fMRI was performed in a block design experiment using a blood oxygenation level-dependent (BOLD)-sensitive echo planar imaging (EPI) sequence (TR $2640 \mathrm{~ms}$, TE $30 \mathrm{~ms}$, flip angle $90^{\circ}, 36$ transverse slices, $0.75 \mathrm{~mm}$ gap, voxel size $3 \times 3 \times 3 \mathrm{~mm}$, base resolution $64 \times 64)$. Point spread function mapping was used for online EPI distortion correction (Zaitsev et al., 2004). Subjects performed a simple visually guided motor task. Instructions were projected on a screen mounted on the rear end of the head coil. A total of 192 scans were acquired while subjects performed four blocks of alternating rest, pursing movements of lips, flexion movements of the fingers of the left hand, fingers of the right hand, toes of the right foot, and toes of the left foot (21 s per condition). Subjects were instructed to perform the movements at a self-paced rate of $\sim 2 \mathrm{~Hz}$. For DTI, a single shot spin-echo EPI with parallel imaging technique GRAPPA (generalized auto-calibrating partially parallel acquisitions) (acceleration factor 2) was used. The sequence was performed with 12 gradient directions and a b-value of 700 (TR $7200 \mathrm{~ms}$, TE $80 \mathrm{~ms}$, voxel size $1.9 \times 2.0 \times 1.9 \mathrm{~mm}, 60$ transverse slices, base resolution $128 \times 128$ ).

fMRI analysis. The fMRI data were processed using Brain Voyager (Brain Innovation, Maastricht, The Netherlands). Before statistical analysis, interslice scan time correction with sinc interpolation and threedimensional (3D) motion correction were used for data preprocessing. No relevant motion artifacts were detected. The realigned time series were temporally filtered with a high-pass filter of three data points. Linear drifts of the signal were removed from each pixel's time course. For alignment of fMRI and 3D anatomical data, a two-stage coregistration process was applied with an initial automatic header-based alignment, followed by fine tuning through a manual adjustment of rotational and translational parameters. At each voxel, a hemodynamic reference function was correlated with the time course of the measured BOLD signal, resulting in a correlation coefficient $r$. The data were thresholded at $80 \%$ of the maximum correlation coefficient in $\mathrm{M} 1$ for a given motor task to obtain circumscribed functional representations of comparable size in M1. The anatomical and fMRI data were exported as DICOM files.

Fiber tracking. FA maps were calculated from the DTI data. T1weighted MPRAGE, fMRI, and DTI were fused (PatXfer 5.1, BrainLab, image fusion software module) by a semiautomatic rigid registration procedure using intensity-based information (Nimsky et al., 2006). Fiber tracking was performed based on a tensor deflection algorithm in which the trend of the current generated fibers is also considered (Lazar et al., 2003; Nimsky et al., 2006). The major eigenvector of each seed was cal- culated, and iteration was started bidirectionally until one of the following stop criteria was met: FA $<0.15$ or deviation angle $>40^{\circ}$. Tracked fibers with a length $<50 \mathrm{~mm}$ were discarded from analysis. Retained fibers were visualized by directionally encoded color (Pajevic and Pierpaoli, 1999) (Figs. 1a-d, 2a).

Fiber tracking was started from a large rectangular ROI that covered the lip, hand, and foot representations defined by fMRI in the right and left M1 (Fig. 1a). This first tracking step visualized corticofugal fibers originating from M1 (Fig. 1a). In a second step, another rectangular ROI was placed within the area of the CC that was identified by the first tracking step, and fiber tracking was performed again (Fig. 1b). Both ROIs were set to contain 30,000 seeds, regardless of their size. Because the BOLD signal is largely limited to the gray matter, tracked fibers projecting into or near to the functionally defined M1 representations were retained, including at least one more voxel beyond the gray/white matter boundary (Fig. $1 c-d$ ). For the CMFs projecting into the M1 hand area, the knob of the precentral gyrus, an accepted anatomical landmark of the M1 hand area (Yousry et al., 1997) was used in addition to define the hand CMF target area (Fig. 1c,d). Tracked CMFs were automatically generated as 3D objects (BrainLab) and color encoded (lip CMFs: red, hand CMFs: green, and foot CMFs: orange) (Fig. 2a). Validity and specificity of this novel two-step tracking procedure were ensured by comparing tracking results with those of a one-step procedure that started from a ROI covering the entire CC as described previously by others (Hofer and Frahm, 2006; Zarei et al., 2006; Johansen-Berg et al., 2007). Both procedures revealed identical anatomical sites of tracked fibers in the CC, but the two-step tracking showed superior sensitivity; i.e., more fibers were tracked.

Image normalization. Images of all subjects were aligned to the anterior commissure (AC)/posterior commissure (PC) line. The aligned individual CMF data were exported in DICOM format and transformed on the basis of a proportional grid principle. Usually linear transformation on the basis of 12 parameters (Fox et al., 1984) matches individual data well on the cortical level, whereas deep internal structures of the brain, such as the CC, are not matched satisfactorily. Therefore, a modified linear transformation (in-house plug-in for Brain Voyager; Steffen Volz, Brain Imaging Center, Johann Wolfgang Goethe-University Frankfurt, Frankfurt am Main, Germany) was performed, where the parameters were defined by the individual CC structure: the interhemispheric plane, the plane through the genu of the CC parallel to AC-PC and planes through AC and branch of fornix (instead of PC). The outer border was determined by the extent of the CC. This allowed warping individual CC data onto the CC morphology of one reference subject (see Figs. 3, 4). The individual transformed CMF data were superimposed to depict interindividual variability of CMF topography (see Fig. 4).

DTI quantification. FA was averaged separately for hand versus foot CMFs from all voxels within the boundaries of the CC rather than from the entire CMF pathway. This restriction was applied to avoid contamination from crossing fibers, which are absent within the midbody of the CC but certainly present in other parts of the subcortical white matter. To avoid inclusion of partial volumes of surrounding CSF, a distance of one voxel to the CC/CSF boundary (indicated by FA $<0.4$ ) was respected (Fig. 1e,f).

IHI between the hand areas of M1. IHI between the hand areas of M1 was measured by an established paired-pulse TMS protocol (Ferbert et al., 1992). TMS was delivered through two figure-of-eight coils (diameter of each loop, $70 \mathrm{~mm}$ ), each connected to a Magstim 200 magnetic stimulator with a monophasic current waveform (Magstim, Dyfed, UK). The conditioning coil was placed tangentially over the hand area of the left M1, with the handle pointing laterally so that a lateral-to-medial current was induced in this M1. The test coil was placed over the hand area of the right $\mathrm{M} 1$, with the handle pointing backwards and $45^{\circ}$ away from the midline so that the induced current in this M1 flowed from lateral/posterior to medial/anterior. For both coils, the optimal position was identified that resulted in largest motor evoked potentials (MEPs) in the first dorsal interosseus (FDI) muscle of the contralateral hand. The resting motor threshold (RMT) was determined for the conditioning pulse to the nearest $1 \%$ of maximum stimulator output and was defined as the minimum stimulus intensity that resulted in liminal MEPs $>50 \mu \mathrm{V}$ in at 
least 5 of 10 trials. IHI increases with the intensity of the conditioning stimulus (Ferbert et al., 1992; Hanajima et al., 2001). Accordingly, the intensity of the conditioning stimulus was varied from 100 to $150 \%$ RMT in $10 \%$ steps (i.e., six different intensities) to obtain a wide range of IHI magnitudes from threshold to maximum. The intensity of the test pulse was adjusted to produce an unconditioned MEP of on average $1 \mathrm{mV}$ in peak-to-peak amplitude. The interstimulus interval between conditioning and test pulse was set to $12 \mathrm{~ms}$, because previous studies showed reliable IHI at this interval (Ferbert et al., 1992; Hanajima et al., 2001). Eight trials per condition were run in randomized order, and conditional averages of the single-trial MEP amplitudes were calculated. The intertrial interval varied randomly between 4 and $6 \mathrm{~s}$. IHI was expressed for each intensity of the conditioning pulse by ( 1 - mean conditioned MEP/ mean unconditioned MEP) $\times 100 \%$. Accordingly, IHI $=0 \%$ indicates no inhibition, and $\mathrm{IHI}=100 \%$ indicates complete inhibition. In addition, IHI threshold was determined for each subject by fitting a linear or second order binomial function to the individual IHI intensity curve and extrapolate those intensities of the conditioning pulse (expressed as a percentage of maximum stimulator output) that resulted in 25 or 50\% IHI (Orth et al., 2003).

MEPs were recorded bilaterally from the FDI by surface electromyography (EMG), with the active electrode placed over the muscle belly and the reference electrode on the metacarpophalangeal joint of the index finger. The EMG was bandpass filtered ( $20 \mathrm{~Hz}$ to $2 \mathrm{kHz}$ ) and amplified (Counterpoint Mk2 Electromyograph, Dantec, 2740 Skovlunde, Denmark), digitized at an analog-to-digital rate of $4 \mathrm{kHz}$ per channel (CED Micro 1401; Cambridge Electronic Design, Cambridge, UK), and stored on a computer for off-line analysis. Customized Spike2 software (Cambridge Electronic Design) was used for data acquisition and analysis.

Statistics. To relate microstructure to functional connectivity, individual mean FA values of the hand CMFs were correlated to IHI (interhemispheric inhibition between the hand areas of M1) and to IHI threshold, using linear regression analyses. To test for topographical specificity, FA values of the foot CMFs were also correlated to the same IHI data. Statistical significance was assumed if $p<0.05$.

\section{Results}

\section{CMF topography and somatotopy}

Figure 3 shows the midsagittal topography of hand CMFs (encoded in green) versus foot CMFs (encoded in orange) of all 12 tested subjects. CMFs mapped consistently onto the posterior body and/or isthmus of the CC. CMFs of the hand and foot overlapped to some extent but showed a consistent somatotopy. In all subjects, the hand CMFs were located slightly more ventrally than the foot CMFs, and in 10 of 12 subjects the hand CMFs were located slightly more anteriorly than the foot CMFs (Fig. 3). Only subjects 7 and 9 showed a partially reverse somatotopy with the hand CMFs located posteriorly to the foot CMFs (Fig. 3). The lip CMFs could be reliably tracked in only one subject (Fig. 2). In this individual, the lip CMFs were located most anteriorly, merg-
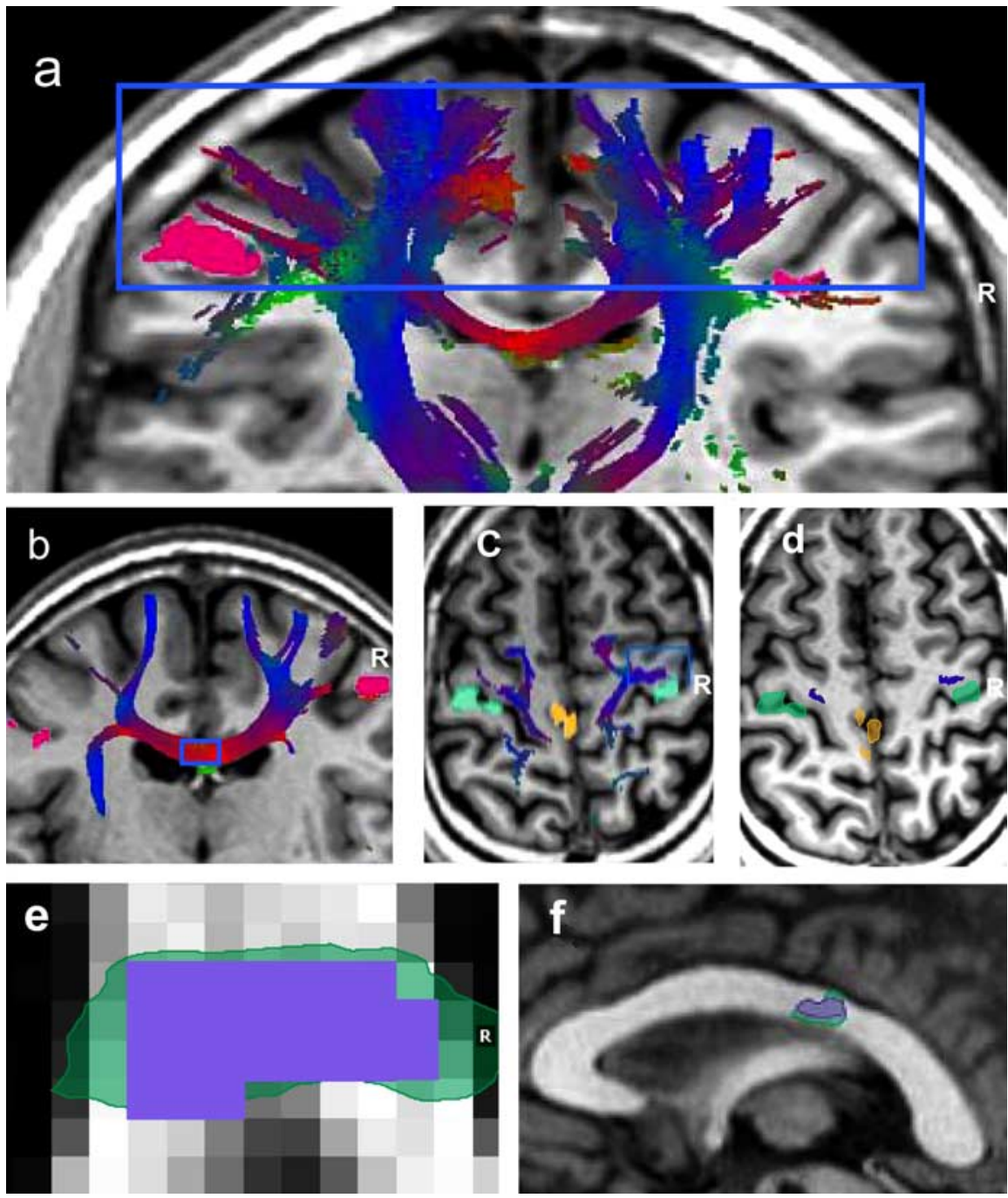

Figure 1. Combined fMRI/DTI procedure to track CMFs. $\boldsymbol{a}$, Tracked fibers after first tracking step: a large ROI (blue rectangle) was set that covered the fMRI-defined M1 face, hand, and foot representations of both hemispheres. $\boldsymbol{b}, A$ second ROI (blue target ROI (blue rectangle) was centered on the knob of the right precentral gyrus, an accepted anatomical landmark for the M1 hand representation (Yousry et al., 1997). The closely adjacent fMRI-defined M1 hand area is depicted in green. $\boldsymbol{d}$, Only those bidirectionally from the seeds in the CC, fibers are retained also in a homologous region of the hand area of the left M1. A similar procedure was performed for the fMRI-defined M1 lip (magenta in $\boldsymbol{a}, \boldsymbol{b}$ ) and foot areas (orange; tracked fibers not shown). $\boldsymbol{e}$, Selected voxels for FA calculation (purple) superimposed on the tracked hand CMFs (green), axial view at high magnification. $\boldsymbol{f}$, Data from the same subject as in $\boldsymbol{e}$, midsagittal view. R, Right.

ing into the anterior body of the CC. Figure 4 shows the interindividual variability of CMF topography by superimposing the individual normalized hand CMFs (Fig. 4a) and foot CMFs (Fig. $4 b$ ) onto one reference midsagittal CC. The arithmetic mean Talairach coordinates of the hand CMFs were $y=-14.3 \pm 3.2 \mathrm{~mm}$ and $z=17.6 \pm 0.8 \mathrm{~mm}$, and those of the foot CMFs were $y=$ $-17.1 \pm 2.8 \mathrm{~mm}$ and $z=18.8 \pm 0.9 \mathrm{~mm}$. Differences were significant for both axes ( $y: p=0.049 ; z: p=0.0005)$, confirming a more anterior and ventral location of the hand CMFs compared with the foot CMFs (Fig. 4c).

\section{Correlation between FA and IHI}

Linear regression analysis revealed a positive correlation between FA of the hand CMFs and the magnitude of IHI, when intensities of the conditioning pulse of $130 \% \operatorname{RMT}(r=0.59 ; p=0.04)$, $140 \% \operatorname{RMT}(r=0.66 ; p=0.02)$, or $150 \% \operatorname{RMT}(r=0.59 ; p=$ 

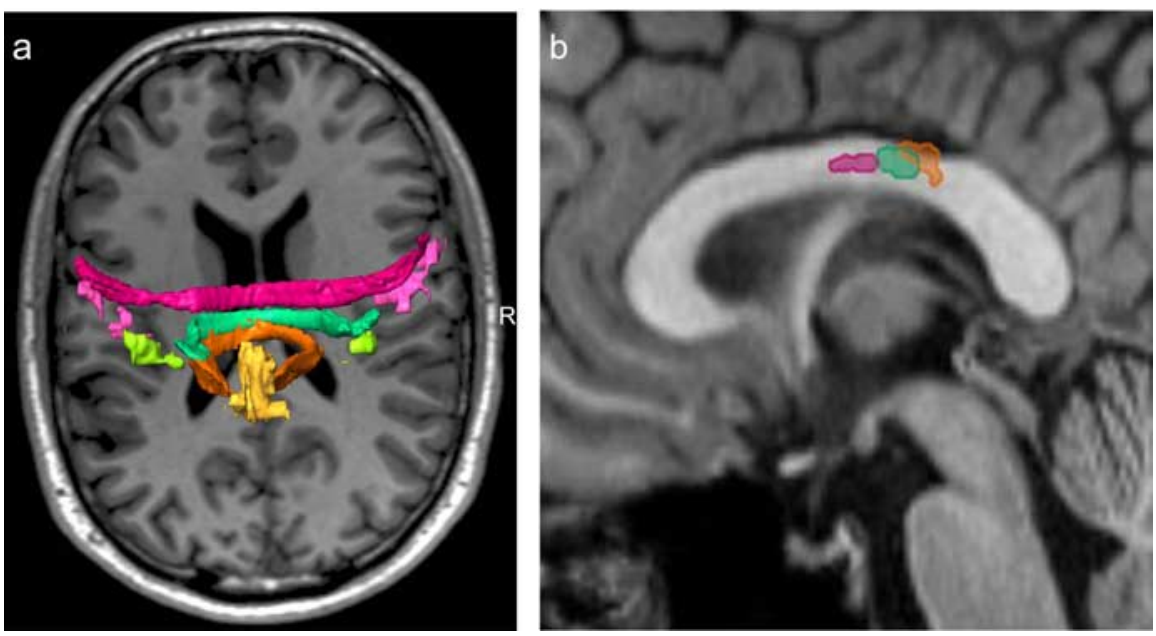

tural information (fractional anisotropy) links to their function (interhemispheric inhibition).

\section{Topography of CMFs}

Largely based on experimental work in monkeys (Pandya et al., 1971; Pandya and Seltzer, 1986; Schmahmann and Pandya, 2006) and a few anatomical studies in humans (van Valkenburg, 1913; de Lacoste et al., 1985), it was long thought that the CMFs connecting M1 of the two hemispheres traverse the human CC in its anterior midbody (Witelson, 1989). However, one TMS study (Meyer et al., 1998) and two recent DTI tractography studies (Hofer and Frahm, 2006; Zarei et al., 2006)

Figure 2. a f fMRI-defined M1 representations (lip: light red; hand: light green; foot: yellow) and tracked CMFs (lip: dark red; hand: dark green; foot: orange) visualized as 3D objects in one subject. $\boldsymbol{b}$, The CMFs (midsagittal view) pass through the posterior body and isthmus of the CC. Note the somatotopic order with lip CMFs located most ventrally and anteriorly, the foot CMFs located most dorsally and posteriorly, and the hand CMFs located in between. $R$, Right.
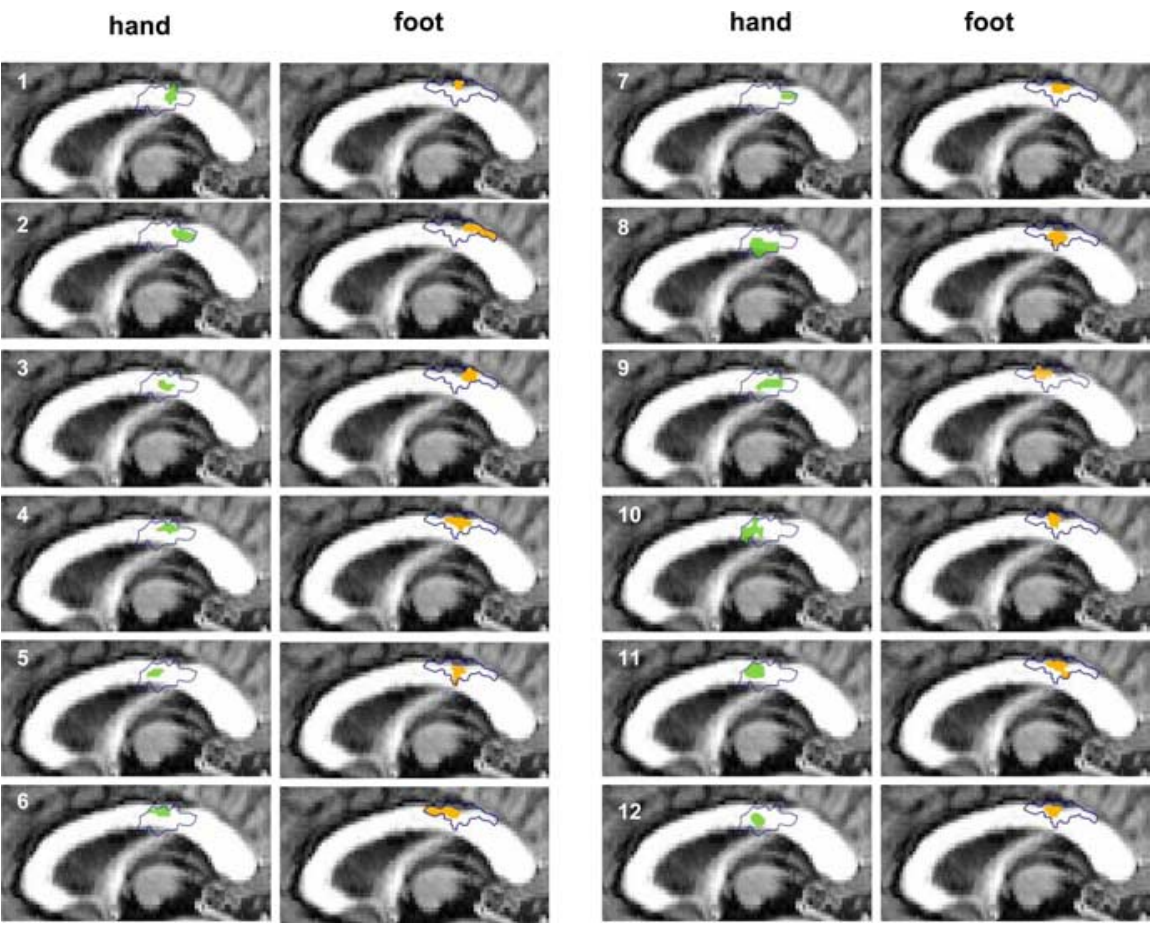
strongly suggested that CMFs cross the human CC clearly more posteriorly, in the posterior body of the CC. Our study confirms this posterior topography and specifies the information from the previous DTI studies by using fMRI in addition to anatomical criteria to define M1 as the tractography target region. One explanation for the posterior shift of CMFs in humans compared with monkeys is the significant increase in prefrontal cortex volume, in particular the prefrontal white matter, in humans (Eccles, 1989; Schoenemann et al., 2005), suggesting connectional elaboration. Indeed, prefrontal cortex heavily connects between hemispheres by callosal fibers, which occupy the complete anterior half of the human CC (Zarei et al., 2006).

It should be noted that the exact topography of CMFs in the present study showed considerable interindividual variability along the anterior-posterior axis (Figs. 3, 4). Coregistration of T1-weighted anatomical images, fMRI, and DTI tractography offers the opportunity to determine where exactly CMFs are localized in the CC of a given individual. The fibertracking results were further validated by demonstrating, according to a previous

Figure 3. Midsagittal topography of hand CMFs (green) and foot CMFs (orange) in all 12 subjects rendered in a modified Talairach space. The blue contours indicate the outlines, if the hand and foot CMFs from all subjects were superimposed on one plot. Note consistent location of the CMFs in the posterior body and isthmus of the $\mathrm{CC}$ in all subjects, and ventral and/or anterior location of the hand CMFs relative to the foot CMFs in all subjects except subject 7 and 9.

0.04) were used (Fig. 5a). In addition, FA of the hand CMFs correlated negatively with the threshold intensities for $25 \%(r=$ $-0.60 ; p=0.04)$ and $50 \%$ IHI $(r=-0.64 ; p=0.02)$ (Fig. $5 b)$. In contrast, FA of the foot CMFs did not correlate with any of these IHI measures (all $p$ values $>0.6$ ) (Fig. $5 c, d$ ).

\section{Discussion}

The main findings from this study are that CMFs map onto a circumscript region in the posterior body and isthmus of the human CC and are somatotopically organized, and microstruc- protocol (Dougherty et al., 2005), a high degree of anatomical convergence in the CC if hand CMFs to the left and right M1 were tracked independently (data not shown). In addition, the tracked hand CMFs originated largely from the hand areas of M1. A significant contribution from other premotor areas, in particular the rostrally adjacent dorsal premotor area, is unlikely because these fibers, although they may partially overlap with the CMFs, extend far into the anterior midbody of the CC (Hofer and Frahm, 2006; Zarei et al., 2006), where no fibers were tracked in the present study.

The consistent findings from now three DTI tractography studies with respect to CMF localization should lead to a shift of the previously popular segmentation schemes of the CC that were based on geometrical principals (de Lacoste et al., 1985; Witelson, 

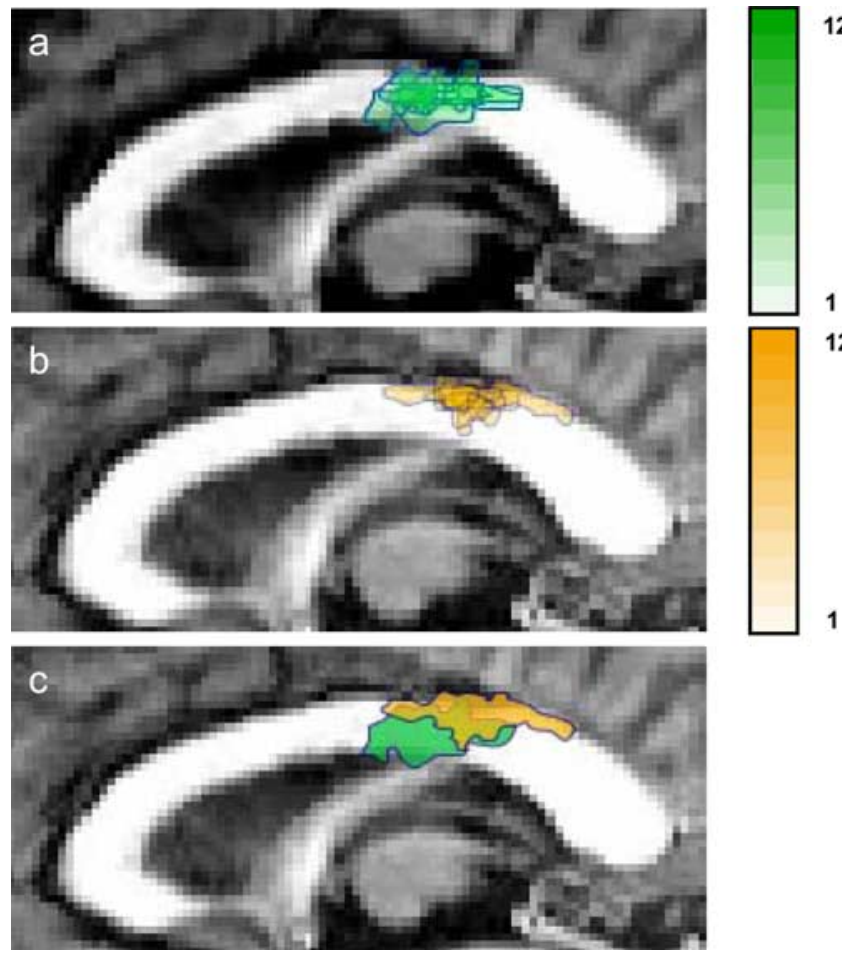

1

Figure 4. $\boldsymbol{a}, \boldsymbol{b}$, Superimposition of the hand CMFs ( $\boldsymbol{a}$ ) and foot CMFs ( $\boldsymbol{b}$ ) of all subjects onto one CC in modified Talairach space. Color bars indicate the number of subjects with CMFs at the same site. $c$, The topographical extent of the superimposed hand CMFs (green) and foot CMFs. Note the separation of hand and foot CMFs along a ventral/anterior-to-dorsal/posterior axis.

1989; Steinmetz et al., 1992; Rajapakse et al., 1996; Jäncke et al., 1997) toward a CC segmentation based on individual anatomical connectivity.

\section{Somatotopy of CMFs}

This is, to the best of our knowledge, the first demonstration that CMFs are somatotopically organized in the human CC. Previous DTI tractography studies identified the topography of CMFs in the human CC but made no effort to subdivide the CMFs into different body representations (Hofer and Frahm, 2006; Zarei et al., 2006). We successfully tracked hand and foot CMFs in all 12 tested subjects and identified a consistent somatotopical order with the hand CMFs situated anteriorly and ventrally from the foot CMFs in 10/12 subjects (Fig. 3). We failed, however, to track lip CMFs except in one subject (Fig. 2). It is unlikely that these fibers do not exist, because previous anatomical tracer studies demonstrated that the M1 face area as defined by intracortical microstimulation is connected with its homolog in the other hemisphere through callosal fibers, at least in the owl monkey (Gould et al., 1986) and in the macaque monkey (Rouiller et al., 1994). Therefore, it is more likely that technical reasons accounted for the failure to track lip CMFs, particularly the well known problem of crossing fibers (Basser et al., 2000; Wiegell et al., 2000; Behrens et al., 2007), which explains the general lack of callosal projections to the lateral surface of the cortex in most DTI studies (Basser et al., 2000; Hofer and Frahm, 2006; JohansenBerg et al., 2007).

Anterograde tract-tracer autoradiography experiments indicated that, similar to the present findings in humans, a somatotopical organization of CMFs also exists in the rhesus monkey with the face CMFs located most anteriorly, sequentially followed more posteriorly by hand and finally foot CMFs (Schmahmann
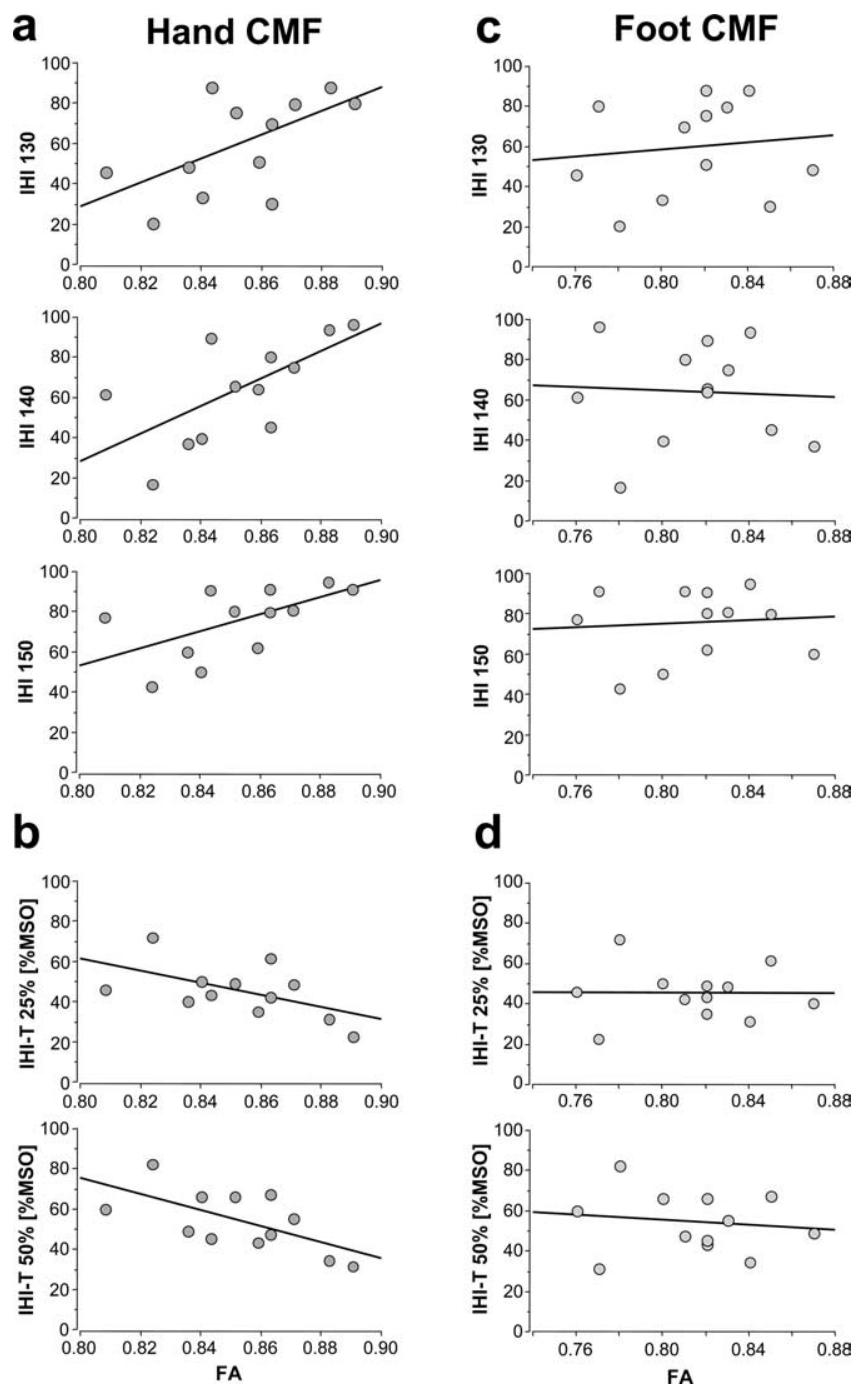

Figure 5. Linkage of microstructure with functional connectivity of the human motor CC. $\boldsymbol{a}$, $\boldsymbol{b}$, Linear regression analysis revealed a positive correlation between the FA of the hand CMFs and the magnitude of $\mathrm{IHI}$ between the hand areas of $\mathrm{M} 1$ at intensities of the conditioning pulse of $130 \% \operatorname{RMT}(r=0.59 ; p=0.04), 140 \% \operatorname{RMT}(r=0.66 ; p=0.02)$, and $150 \%$ RMT $(r=0.59$; $p=0.04)(\boldsymbol{a})$ and a negative correlation with the threshold intensity (IHI-T) of the conditioning pulse [given as a percentage of maximum stimulator output (\%MSO)] to produce $25 \% \mathrm{IHI}(r=$ $-0.60 ; p=0.04)$ and $50 \% \mathrm{IHI}(r=-0.64 ; p=0.02)(\boldsymbol{b})$. Each point reflects $\mathrm{FA}$ and $\mathrm{IHI}$ from one individual subject. $\boldsymbol{c}, \boldsymbol{d}$, This correlation was absent between the FA of the foot CMFs and the $\mathrm{IHI}(\boldsymbol{c})$ and IHI threshold $(\boldsymbol{d})$ between the hand areas of $\mathrm{M} 1$ ( $p$ values $>0.6$ at all intensities of the conditioning pulse).

and Pandya, 2006). This strongly suggests that the present findings are not artificial, because they are substantiated by reliable anatomical techniques.

\section{Correlation between fractional anisotropy and interhemispheric inhibition}

We show for the first time that microstructural information of the hand CMFs as reflected by FA correlated significantly with functional connectivity indexed by IHI between the M1 hand areas in the two hemispheres (Fig. $5 a, b$ ). The biological basis of FA is not entirely clear. This measure is influenced by the degree of myelination, axon size, and axon density in a complex way (Moseley et al., 1990; Beaulieu, 2002; Le Bihan, 2003), but fiber path geometry and crossing fibers may also contribute. In the midbody of the CC, the latter two factors are not relevant because 
fibers run straight and crossing fibers are absent. Light- and electron-microscopic analysis of the fiber composition in the human CC revealed clear regional differences (Aboitiz et al., 1992): thin, largely unmyelinated and densely packed fibers are present preponderantly in the genu and splenium of the CC. In contrast, larger-diameter, myelinated and less densely packed fibers are concentrated in the posterior midbody of the CC (i.e., the CMF region). This regional differentiation of fiber types and densities is paralleled by a regional differentiation of FA, with higher values where thin densely packed CC fibers predominate (Hofer and Frahm, 2006). This suggests that FA in the CC reflects primarily fiber density rather than the degree of myelination or axon diameter. Furthermore, patients with multiple sclerosis showed a direct correlation between FA and CC cross-sectional area (Cader et al., 2007). Because, in a postmortem analysis, patients with multiple sclerosis also exhibited a direct correlation between axon density and CC cross-sectional area (Evangelou et al., 2000), this supports further the notion that FA in the CC is significantly influenced by axon density. This is excellently compatible with the observed correlation between FA and IHI, because it would be expected that more and/or more densely packed CMFs result in stronger IHI and lower IHI threshold.

IHI is thought to be mediated by excitatory transcallosal fibers that originate from the hand area of the conditioning M1 and project onto local inhibitory interneurons in the hand $\mathrm{M} 1$ of the other hemisphere (Ferbert et al., 1992; Di Lazzaro et al., 1999; Hanajima et al., 2001). IHI plays a role in suppressing unwanted mirror movements during intended unimanual movements by inhibiting the "mirror M1" (Hübers and Ziemann, 2006; Duque et al., 2007), and in facilitating unimanual movements by disinhibiting the "active M1" (Duque et al., 2007). Consequently, IHI at rest and its modulation by hand movement are abnormal in patients with acquired mirror movements (Li et al., 2007), and for the paretic hand in stroke patients (Murase et al., 2004; Duque et al., 2005). These data support the notion that IHI reflects functional connectivity that is important in bimanual and unimanual motor control.

One other very recent study also linked microstructure (FA) and a paired-pulse TMS measure of functional corticocortical connectivity, but explored the projection from dorsal premotor cortex to contralateral M1 (Boorman et al., 2007), a network involved in externally cued action selection. It was found that individual differences in FA of white-matter interconnecting regions including dorsal premotor cortex and M1 correlated highly specifically with the TMS-indexed functional connectivity between these two regions when measured during action selection. Finally, correlations between FA and TMS measures of functional connectivity were demonstrated along another important white matter fiber bundle, the corticospinal tract. Patients with diffuse traumatic axonal brain injury showed a positive correlation of FA with MEP amplitude (Yasokawa et al., 2007), and patients with amyotrophic lateral sclerosis, a neurodegenerative disease of the upper and lower motor neuron, exhibited a negative correlation of FA with the central motor conduction time (Sach et al., 2004). These findings and the present results strongly suggest that FA reflects valuable microstructural information that can be linked to electrophysiological measures of functional connectivity in a meaningful way. Potentially, this linkage between structure and function will open up a new and completely noninvasive avenue to understand better the functional relevance of fiber pathways in the human brain.

Finally, we would like to point out that the correlation between FA of the hand CMFs and the magnitude and threshold of
IHI was topographically specific, because it was not seen if FA of the neighboring and even overlapping foot CMFs were correlated with IHI between the hand areas of M1 (Fig. $5 c, d$ ). This lends further credit to the reliability of the techniques used here, and to the perspective that correlations between measures of microstructural information and functional connectivity are not global but rather specific to the system under investigation.

\section{References}

Aboitiz F, Scheibel AB, Fisher RS, Zaidel E (1992) Fiber composition of the human corpus callosum. Brain Res 598:143-153.

Andres FG, Mima T, Schulman AE, Dichgans J, Hallett M, Gerloff C (1999) Functional coupling of human cortical sensorimotor areas during bimanual skill acquisition. Brain 122:855-870.

Basser PJ, Pajevic S, Pierpaoli C, Duda J, Aldroubi A (2000) In vivo fiber tractography using DT-MRI data. Magn Reson Med 44:625-632.

Beaulieu C (2002) The basis of anisotropic water diffusion in the nervous system-a technical review. NMR Biomed 15:435-455.

Behrens TE, Berg HJ, Jbabdi S, Rushworth MF, Woolrich MW (2007) Probabilistic diffusion tractography with multiple fiber orientations: what can we gain? NeuroImage 34:144-155.

Boorman ED, O'Shea J, Sebastian C, Rushworth MF, Johansen-Berg H (2007) Individual differences in white-matter microstructure reflect variation in functional connectivity during choice. Curr Biol 17:1426-1431.

Cader S, Johansen-Berg H, Wylezinska M, Palace J, Behrens TE, Smith S, Matthews PM (2007) Discordant white matter $N$-acetylasparate and diffusion MRI measures suggest that chronic metabolic dysfunction contributes to axonal pathology in multiple sclerosis. NeuroImage 36:19-27.

Caille S, Sauerwein HC, Schiavetto A, Villemure JG, Lassonde M (2005) Sensory and motor interhemispheric integration after section of different portions of the anterior corpus callosum in nonepileptic patients. Neurosurgery 57:50-59; discussion 50-59.

Conturo TE, Lori NF, Cull TS, Akbudak E, Snyder AZ, Shimony JS, McKinstry RC, Burton H, Raichle ME (1999) Tracking neuronal fiber pathways in the living human brain. Proc Natl Acad Sci USA 96:10422-10427.

de Lacoste MC, Kirkpatrick JB, Ross ED (1985) Topography of the human corpus callosum. J Neuropathol Exp Neurol 44:578-591.

Di Lazzaro V, Oliviero A, Profice P, Insola A, Mazzone P, Tonali P, Rothwell JC (1999) Direct demonstration of interhemispheric inhibition of the human motor cortex produced by transcranial magnetic stimulation. Exp Brain Res 124:520-524.

Dougherty RF, Ben-Shachar M, Bammer R, Brewer AA, Wandell BA (2005) Functional organization of human occipital-callosal fiber tracts. Proc Natl Acad Sci USA 102:7350-7355.

Duque J, Hummel F, Celnik P, Murase N, Mazzocchio R, Cohen LG (2005) Transcallosal inhibition in chronic subcortical stroke. NeuroImage 28:940-946.

Duque J, Murase N, Celnik P, Hummel F, Harris-Love M, Mazzocchio R, Olivier E, Cohen LG (2007) Intermanual differences in movementrelated interhemispheric inhibition. J Cogn Neurosci 19:204-213.

Eccles JC (1989) Evolution of the brain: creation of the self. London: Routledge.

Eliassen JC, Baynes K, Gazzaniga MS (1999) Direction information coordinated via the posterior third of the corpus callosum during bimanual movements. Exp Brain Res 128:573-577.

Eliassen JC, Baynes K, Gazzaniga MS (2000) Anterior and posterior callosal contributions to simultaneous bimanual movements of the hands and fingers. Brain 123:2501-2511.

Evangelou N, Esiri MM, Smith S, Palace J, Matthews PM (2000) Quantitative pathological evidence for axonal loss in normal appearing white matter in multiple sclerosis. Ann Neurol 47:391-395.

Ferbert A, Priori A, Rothwell JC, Day BL, Colebatch JG, Marsden CD (1992) Interhemispheric inhibition of the human motor cortex. J Physiol (Lond) 453:525-546.

Fox PT, Perlmutter JS, Raichle ME (1984) Stereotactic method for determining anatomical localization in physiological brain images. J Cereb Blood Flow Metab 4:634.

Gazzaniga MS (2000) Cerebral specialization and interhemispheric communication: does the corpus callosum enable the human condition? Brain 123:1293-1326. 
Gould HJD, Cusick CG, Pons TP, Kaas JH (1986) The relationship of corpus callosum connections to electrical stimulation maps of motor, supplementary motor, and the frontal eye fields in owl monkeys. J Comp Neurol 247:297-325.

Hanajima R, Ugawa Y, Machii K, Mochizuki H, Terao Y, Enomoto H, Furubayashi T, Shiio Y, Uesugi H, Kanazawa I (2001) Interhemispheric facilitation of the hand motor area in humans. J Physiol (Lond) 531:849-859.

Hofer S, Frahm J (2006) Topography of the human corpus callosum revisited-comprehensive fiber tractography using diffusion tensor magnetic resonance imaging. NeuroImage 32:989-994.

Hübers A, Ziemann U (2006) Interhemispheric inhibition and mirror movements [Abstract]. Klin Neurophysiol 37:46.

Innocenti GM (1986) General organization of the callosal connections in the cerebral cortex. In: Cerebral cortex (Jones EG, Peters A, eds), pp 291-353. New York: Plenum.

Jäncke L, Staiger JF, Schlaug G, Huang Y, Steinmetz H (1997) The relationship between corpus callosum size and forebrain volume. Cereb Cortex 7:48-56.

Jeeves MA, Silver PH, Jacobson I (1988) Bimanual co-ordination in callosal agenesis and partial commissurotomy. Neuropsychologia 26:833-850.

Johansen-Berg H, Della-Maggiore V, Behrens TE, Smith SM, Paus T (2007) Integrity of white matter in the corpus callosum correlates with bimanual co-ordination skills. NeuroImage 36 [Suppl 2]:T16-T21.

Kim DS, Kim M (2005) Combining functional and diffusion tensor MRI. Ann N Y Acad Sci 1064:1-15.

Klingberg T, Hedehus M, Temple E, Salz T, Gabrieli JD, Moseley ME, Poldrack RA (2000) Microstructure of temporo-parietal white matter as a basis for reading ability: evidence from diffusion tensor magnetic resonance imaging. Neuron 25:493-500.

Koch MA, Norris DG, Hund-Georgiadis M (2002) An investigation of functional and anatomical connectivity using magnetic resonance imaging. NeuroImage 16:241-250.

Lazar M, Weinstein DM, Tsuruda JS, Hasan KM, Arfanakis K, Meyerand ME, Badie B, Rowley HA, Haughton V, Field A, Alexander AL (2003) White matter tractography using diffusion tensor deflection. Hum Brain Mapp 18:306-321.

Le Bihan D (2003) Looking into the functional architecture of the brain with diffusion MRI. Nat Rev Neurosci 4:469-480.

Le Bihan D, Mangin JF, Poupon C, Clark CA, Pappata S, Molko N, Chabriat H (2001) Diffusion tensor imaging: concepts and applications. J Magn Reson Imaging 13:534-546.

Li JY, Espay AJ, Gunraj CA, Pal PK, Cunic DI, Lang AE, Chen R (2007) Interhemispheric and ipsilateral connections in Parkinson's disease: relation to mirror movements. Mov Disord 22:813-821.

Meyer B-U, Röricht S, Woiciechowsky C (1998) Topography of fibers in the human corpus callosum mediating interhemispheric inhibition between the motor cortices. Ann Neurol 43:360-369.

Moseley ME, Cohen Y, Kucharczyk J, Mintorovitch J, Asgari HS, Wendland MF, Tsuruda J, Norman D (1990) Diffusion-weighted MR imaging of anisotropic water diffusion in cat central nervous system. Radiology 176:439-445.

Murase N, Duque J, Mazzocchio R, Cohen LG (2004) Influence of interhemispheric interactions on motor function in chronic stroke. Ann Neurol 55:400-409.

Nimsky C, Ganslandt O, Fahlbusch R (2006) Implementation of fiber tract navigation. Neurosurgery 58:ONS-292-ONS-303; discussion ONS-303-ONS-304.

Orth M, Snijders AH, Rothwell J (2003) The variability of intracortical inhibition and facilitation. Clin Neurophysiol 114:2362-2369.

Pajevic S, Pierpaoli C (1999) Color schemes to represent the orientation of anisotropic tissues from diffusion tensor data: application to white matter fiber tract mapping in the human brain. Magn Reson Med 42:526-540.

Pandya DN, Seltzer B (1986) The topography of commissural fibers. In:
Two hemispheres - one brain: functions of the corpus callosum (Lepore F, Ptito M, Jasper HH, eds), pp 47-73. New York: Liss.

Pandya DN, Karol EA, Heilbronn D (1971) The topographical distribution of interhemispheric projections in the corpus callosum of the rhesus monkey. Brain Res 32:31-43.

Pfefferbaum A, Adalsteinsson E, Sullivan EV (2003) Replicability of diffusion tensor imaging measurements of fractional anisotropy and trace in brain. J Magn Reson Imaging 18:427-433.

Preilowski BF (1972) Possible contribution of the anterior forebrain commissures to bilateral motor coordination. Neuropsychologia 10:267-277.

Rajapakse JC, Giedd JN, Rumsey JM, Vaituzis AC, Hamburger SD, Rapoport JL (1996) Regional MRI measurements of the corpus callosum: a methodological and developmental study. Brain Dev 18:379-388.

Rouiller EM, Babalian A, Kazennikov O, Moret V, Yu XH, Wiesendanger M (1994) Transcallosal connections of the distal forelimb representations of the primary and supplementary motor cortical areas in macaque monkeys. Exp Brain Res 102:227-243.

Sach M, Winkler G, Glauche V, Liepert J, Heimbach B, Koch MA, Buchel C, Weiller C (2004) Diffusion tensor MRI of early upper motor neuron involvement in amyotrophic lateral sclerosis. Brain 127:340-350.

Schmahmann JD, Pandya DN (2006) Corpus callosum. In: Fiber pathways of the brain (Schmahmann JD, Pandya DN, eds), pp 485-496. New York: Oxford UP.

Schoenemann PT, Sheehan MJ, Glotzer LD (2005) Prefrontal white matter volume is disproportionately larger in humans than in other primates. Nat Neurosci 8:242-252.

Schulte T, Sullivan EV, Muller-Oehring EM, Adalsteinsson E, Pfefferbaum A (2005) Corpus callosal microstructural integrity influences interhemispheric processing: a diffusion tensor imaging study. Cereb Cortex 15:1384-1392.

Steinmetz H, Jäncke L, Kleinschmidt A, Schlaug G, Volkmann J, Huang Y (1992) Sex but no hand difference in the isthmus of the corpus callosum. Neurology 42:749-752.

Tuch DS, Salat DH, Wisco JJ, Zaleta AK, Hevelone ND, Rosas HD (2005) Choice reaction time performance correlates with diffusion anisotropy in white matter pathways supporting visuospatial attention. Proc Natl Acad Sci USA 102:12212-12217.

van Valkenburg CT (1913) Experimental and pathologico-anatomical researches on the corpus callosum. Brain 36:119-165.

Wiegell MR, Larsson HB, Wedeen VJ (2000) Fiber crossing in human brain depicted with diffusion tensor MR imaging. Radiology 217:897-903.

Wiesendanger M, Serrien DJ (2004) The quest to understand bimanual coordination. Prog Brain Res 143:491-505.

Witelson SF (1989) Hand and sex differences in the isthmus and genu of the human corpus callosum. A postmortem morphological study. Brain 112:799-835.

Wolbers T, Schoell ED, Buchel C (2006) The predictive value of white matter organization in posterior parietal cortex for spatial visualization ability. NeuroImage 32:1450-1455.

Yasokawa YT, Shinoda J, Okumura A, Nakayama N, Miwa K, Iwama T (2007) Correlation between diffusion-tensor magnetic resonance imaging and motor-evoked potential in chronic severe diffuse axonal injury. J Neurotrauma 24:163-173.

Yousry T, Schmid U, Alkadhi H, Schmidt D, Peraud A, Buettner A, Winkler P (1997) Localization of the motor hand area to a knob on the precentral gyrus. Brain 120:141-157.

Zaitsev M, Hennig J, Speck O (2004) Point spread function mapping with parallel imaging techniques and high acceleration factors: fast, robust, and flexible method for echo-planar imaging distortion correction. Magn Reson Med 52:1156-1166.

Zarei M, Johansen-Berg H, Smith S, Ciccarelli O, Thompson AJ, Matthews PM (2006) Functional anatomy of interhemispheric cortical connections in the human brain. J Anat 209:311-320. 